\title{
Views of Maternity Nurses Relating to Barriers in Early Initiation of Breastfeeding: A Qualitative Study
}

\author{
(D) Sibel Serap Ceylan, @ Bengü Çetinkaya \\ Pamukkale University Faculty of Health Science, Department of Pediatric Nursing, Denizli, Turkey
}

\begin{abstract}
Aim: Although breastfeeding is common in Turkey, the proportion of breastfed infants in the first hour after birth is not at the desired level. The aim of this study was to explore the difficulties in the early initiation of breastfeeding as encountered by maternity nurses.

Materials and Methods: A phenomenology model of qualitative research design was used in this study. The data were collected by an in-depth interview method. Content analysis was used to analyze the data, and findings were categorized into themes.

Results: Barriers related to the early initiation of breastfeeding was grouped into four main themes: Delivery, culture, hospital environment and health care staff. The delivery mode, pain and the perception of insufficient milk were included in the theme of 'delivery'. The theme of 'culture' consisted of traditional practices, patriarchal family structure, and language sub-themes. The theme of 'hospital environment' involved lack of privacy and chaos. Finally understaffing, insufficient knowledge and employee turnover rates were included in the 'health care staff' theme.

Conclusion: Interviews with maternity nurses about barriers to the early initiation of breastfeeding provided deeper understanding into this critical period. These findings suggest that delivery related factors and cultural and environmental elements were obstacles in initiating early breastfeeding. Support and counseling given in the early postpartum period is important for the initiation of breastfeeding. To eliminate the barriers in this process, it is recommended to improve training and health care policy.
\end{abstract}

Keywords: Breastfeeding, breastfeeding initiation, breastfeeding barriers, nurse, qualitative research

\section{Introduction}

Breastfeeding is fundamental for an infant's survival, nutrition, growth and development, and maternal health. Early initiation of breastfeeding, defined by the World Health Organization (WHO) as giving mother's first milk, known as colostrum, to infants within the first hour of birth, provides protective factors against illnesses. The $\mathrm{WHO}$ recommends that children initiate breastfeeding within the first hour of birth and be exclusively breastfed for the first 6 months of life, followed by continued breastfeeding with suitable supplementary foods for up to two years or beyond. In 2012, a universal practice plan on maternal, infant and young child nutrition specifying six global nutrition targets for 2025 was endorsed. One of these targets was to increase the rate of exclusive breastfeeding in the first six months up to at least $50 \%$. Unfortunately, breastfeeding practices are not at a desirable level throughout the world. Currently, the global average of exclusively breast-fed infants under 6 months is only 37\% (1). The United Nations Children's Fund (UNICEF) indicate that most of the world's newborns are waiting too long to begin breastfeeding, and in 2017 alone, an estimated 78 million newborns had to wait more than 1 hour to begin breastfeeding (2).

The WHO and UNICEF published a joint statement in 1989 on "Protecting, promoting and supporting breastfeeding: the special role of maternity services", in 
order to support mothers and optimize the opportunity of breastfeeding in line with its recommendations. This statement listed "Ten Steps to Successful Breastfeeding". Afterwards, the WHO published the Baby-friendly Hospital Initiative in 1991 and updated it in 2009 (1,3). Since 1991, the "Promotion of Breastfeeding and Baby-friendly Health Facilities Program" which aims to protect, encourage and support breastfeeding has been carried out in Turkey. This program, which follows the recommendations of the $\mathrm{WHO}$, promotes the key message that babies should start breastfeeding immediately after birth and only be breastfed for the first six months, with breastfeeding continuing until the age of two supplemented by appropriate food after the first six months (4). Breastfeeding is quite common in Turkey. According to the results of the Turkish Demographic and Health Survey 2013, 96.4\% of babies were fed with breast milk for at least a while. However, only $49.9 \%$ of babies started to breastfeed within the first hour after birth. The rate of other liquids given instead of breastmilk is $25.7 \%$ three days after birth. $57.9 \%$ of babies are fed exclusively by breastfeeding until the second month. The rate of exclusively breastfed babies until the fourth month is $35.4 \%$. This rate decreases rapidly with the child's age, and the proportion of bottle users increases with age (5). Also, in 2017 the Turkey Health Statistics Report, it was reported that $30.8 \%$ of infants between 0-6 months and 32\% of infants between 7-12 months are breastfed (6). Although breastfeeding is common in Turkey, exclusively breastfed rates are not at the desired level.

Early initiation is important for the effectiveness and continuity of breastfeeding. Early breastfeeding has a positive effect on lactation, uterine involution, motherinfant interaction and the baby's development. The successful initiation of breastfeeding concerns the events and practices during labor, birth and the immediate postbirth period (7). For the initiation of breastfeeding within the first hour of life, mothers need adequate support, direction and encouragement on the positioning and breastfeeding of their newborns (8).

Causes such as the mother's knowledge of the benefits and skills of correct breastfeeding techniques, the mother's social, cultural, and economic status and support systems (particularly the family and health system personnels' skills) affect the initiation of breastfeeding (7). For example, there are some cultural practices such as feeding newborns supplemental foods or drinks by an elderly family member (8). Pre-lacteal feeding is defined as the administration of any foods or liquids other than breast milk to an infant before the initiation of breastfeeding and it can delay the initiation of breastfeeding. The practice of offering pre-lacteal feedings to a newborn is reported as an early mechanism for the disruption of exclusive breastfeeding (1). Other factors and reasons for missing out on breastfeeding in the first hour of life are lack of knowledge, caesarean delivery, and immediate separation of mothers and babies after birth (8).

Starting and maintaining a successful breastfeeding process is influenced by many factors. To ensure the success of lactation, the support of nurses in the early postpartum period is important. Resolving problems related to breastfeeding is one of the tasks of nurses. However, few qualitative studies have been conducted that specifically addressed the experiences of nurses regarding breastfeeding. Understanding the experiences of maternity nurses may help healthcare professionals to prepare education programs and care guides that enhance the quality of nursing care. In addition, these programs and guides may help healthcare professionals to recognize the early detection of breastfeeding barriers and support women to overcome these barriers in the early postpartum period. Therefore, this study was conducted to determine the difficulties in the early initiation of breastfeeding encountered by nurses working in maternity clinics.

\section{Materials and Methods}

\section{Design}

We used a phenomenological model of qualitative research design in this study.

\section{Setting and Participants}

We collected data between June and December 2018 in a university and two state hospitals. The hospitals were Baby-friendly Health Initiative accredited. The participants were nurses working in maternity clinics. The inclusion criteria were as follows: Working in a maternity clinic for a minimum of six months, voluntary participation, experience with breastfeeding counseling.

We used a purposive sampling method to select nurses in the study. There were 18 nurses working at the maternity clinics. Data collection continued until saturation had been achieved and no new themes were emerging (9). The study was completed with thirteen nurses.

\section{Data Collection}

We obtained the maternity nurses' work schedule from the chief nurse, went to the clinic diurnally and determined those nurses who met the study's sample selection criteria. We informed nurses in the study group about the 
study purpose and obtained their consent. We made an appointment with the maternity nurses for the end of their shift. We collected data by the in-depth interview method in the clinic's meeting room. The interviews with nurses were conducted individually using a semi-structured interview form. The interviews were tape-recorded and additional written notes were taken.

The interview started with meeting and informing the participant. First, those questions on the participant's descriptive characteristics form were asked. Then, the interview continued with the first question on the semistructured interview form: "What are the barriers you have to the early initiation of breastfeeding at the clinic?". During the interview, the researchers let participants speak freely and discuss opinion and thoughts. Interviews were varied in time, they lasted between 38 and 52 minutes.

We used a participant descriptive characteristics form and semi-structured interview form to collect the data. The participant descriptive characteristics form included questions on socio-demographic properties such as age, gender, education level, work experience, having children and breastfeeding status. As part of the qualitative research method, data were collected by a semi-structured interview form (Table I). It was developed based on the purposes of the study in light of the relevant literature (10-12).

\section{Statistical Analysis}

Data analysis was performed by the researchers. Sociodemographic variables were expressed as means and numbers.

We evaluated qualitative data by content analysis. According to this procedure, firstly one researcher transcribed the interviews directly into the computer without any changes. While listening to the tape recordings "who said what" was noted. Next, two researchers read the written interviews a few times individually to find out about them generally. After reading, these researchers disintegrated the text into meaningful parts, separated the content, and each significative part was attached with a code. Then,

Table I. Semistructured interview form

\section{Questions}

1. What are the barriers you have with the early initiation of breastfeeding at the clinic?

2. What are the obstacles caused by delivery related factors in the early initiation of breastfeeding?

3. What are the obstacles caused by cultural factors in the early initiation of breastfeeding?

4. What are the obstacles caused by the hospital environment in the early initiation of breastfeeding? these codes were compared with regard to their similarities and differences and organized into categories. Finally, we classified and reported the data. After the classification process, themes were identified and named (9).

We worked individually and independently from one another within these processes. Afterwards, we discussed the thematic statements and came to an agreement on the themes which described the results best.

\section{Trustworthiness}

The trustworthiness criteria in qualitative research are credibility, transferability, dependability and confirmability (9).

The first researcher conducted the interviews, and the second researcher observed and recorded the nurses' behavior in order to enhance study trustworthiness. In order to support the honesty of the participants, the nurses who volunteered to participate in the study were included in the study and it was explained that they could withdraw from the study without giving any reason. It was also stated that there were no correct answers to the questions asked to them. Furthermore, peer debriefing and member checking were used to enhance credibility. To ensure transferability of the study, purposive sampling was used. Also, during the interviews, a tape-recorder was used and additional written notes were taken. After the interviews, the recordings were copied word for word, the facial/bodily expressions of nurses were described by two of the researchers independently without additional interpretation as to their intent or meaning. For consistency, the data were analyzed independently by two researchers. Researchers resolved differences between the findings by consensus, and arranged and documented the results. For confirmability, an expert evaluated the comprehensive interview form and the final version of the thematization.

\section{Ethical Considerations}

Approval of the Ethics Committee and permission of the institution were taken (decision no: 60116787-020/29033). Verbal and written permission was obtained from the participants before the interviews commenced and were tape-recorded. In the presentation of the results, participant comments remain anonymous.

\section{Results}

\section{Demographic Information}

A total of 13 maternity nurses were included in this study. In the current study, all of the participants were female and ranged in age from 32 to 48 years. All of the participants were married, had at least one child, and all of 
them gave their children breast-milk for at least 6 months. Five of the nurses had undergraduate degrees. Four had associate degrees, and four were high school graduates. The mean working years of their maternity nursing was 9.15 years. All of them were trained about breastfeeding consultancy (Table II).

The findings taken from the interviews were classified into four main themes: (1) delivery, (2) culture, (3) hospital environment, and (4) health care staff (Table III).

\section{Delivery}

This theme was investigated through three subthemes; delivery mode, pain and perception of insufficient milk.

\section{Delivery Mode}

Most of the participants expressed their belief that the delivery mode had an effect on the initiation of breastfeeding. According to the statements of the participants, cesarean delivery negatively affected the baby's first breastfeeding time, the mother's breastfeeding skill and the baby's sucking skill. In particularly, they noted: "Breastfeeding skills in cesarean birth are related to us and the sitter... Her (the mother) breastfeeding skill is passive... She is dependent on us... It is hard to get into position for breastfeeding after the mother starts to come to herself..." (N4).

\section{Pain}

The participants stated that mothers did not want to breastfeed because of pain after birth: "...she does not want to breastfeed because she is in pain..." (N8).

\section{Perception of Insufficient Milk}

All of the participants indicated that the mothers thought their breastmilk volume to be insufficient right after birth, especially in the case of a cesarean delivery. Here is a typical comment: "... In general, it is known among the people that lactation is late... In fact, the mother has milk, but the baby cannot feed, because it does not suck properly..."(N7).

\section{Culture}

Cultural barriers to the initiation of breastfeeding were traditional practices, patriarchal family structure and language.

Table III. Themes and subthemes of obstacles to breastfeeding initiation

\begin{tabular}{|l|l|}
\hline \multirow{4}{*}{ Themes } & Subthemes \\
\hline \multirow{4}{*}{ 2. Culture } & Delivery mode \\
\cline { 2 - 2 } & Pain \\
\cline { 2 - 2 } & Perception of insufficient milk \\
\hline \multirow{3}{*}{ 3. Hospital environment } & Traditional practices \\
\cline { 2 - 2 } & Patriarchal family structure \\
\hline \multirow{4}{*}{ 4. Health care staff } & Language \\
\hline & Lack of privacy \\
\cline { 2 - 2 } & Chaos \\
\hline & Understaffing \\
\cline { 2 - 2 } & Insufficient knowledge \\
\cline { 2 - 2 } & Employee turn rates \\
\hline
\end{tabular}

\begin{tabular}{|c|c|c|c|c|c|c|c|c|}
\hline $\begin{array}{l}\text { Participants } \\
\text { no }\end{array}$ & Age & Gender & $\begin{array}{l}\text { Marital } \\
\text { status }\end{array}$ & Education level & $\begin{array}{l}\text { Situation } \\
\text { to have } \\
\text { children }\end{array}$ & $\begin{array}{l}\text { Breastfeeding } \\
\text { status }\end{array}$ & $\begin{array}{l}\text { Work experience } \\
\text { in maternity clinic } \\
\text { (year) }\end{array}$ & $\begin{array}{l}\text { Breastfeeding } \\
\text { consultancy } \\
\text { education }\end{array}$ \\
\hline N1 & 40 & Female & Married & High school & Yes & Yes & 13 & Yes \\
\hline N2 & 44 & Female & Married & Undergraduate & Yes & Yes & 3 & Yes \\
\hline N3 & 47 & Female & Married & High school & Yes & Yes & 5 & Yes \\
\hline N4 & 41 & Female & Married & High school & Yes & Yes & 9 & Yes \\
\hline N5 & 38 & Female & Married & Associate degree & Yes & Yes & 4 & Yes \\
\hline N6 & 48 & Female & Married & High school & Yes & Yes & 4 & Yes \\
\hline N7 & 42 & Female & Married & Associate degree & Yes & Yes & 16 & Yes \\
\hline N8 & 43 & Female & Married & Associate degree & Yes & Yes & 12 & Yes \\
\hline N9 & 39 & Female & Married & Undergraduate & Yes & Yes & 8 & Yes \\
\hline N10 & 35 & Female & Married & Associate degree & Yes & Yes & 11 & Yes \\
\hline N11 & 32 & Female & Married & Undergraduate & Yes & Yes & 10 & Yes \\
\hline $\mathrm{N} 12$ & 38 & Female & Married & Undergraduate & Yes & Yes & 15 & Yes \\
\hline N13 & 36 & Female & Married & Undergraduate & Yes & Yes & 9 & Yes \\
\hline
\end{tabular}




\section{Traditional Practices}

The participants stated that traditional practices, such as giving the baby sherbet and putting a date in the baby's mouth, prevented the first food from being breast milk and caused problems in the initiation of breastfeeding. For example: "... The other day, I trained a mother about breastfeeding. After a while, I went back to the patient's room. There was something dark around the child's mouth. I think it was a date. I panicked thinking it was blood at first. Then I touched something sticky. Then I looked around... They had soaked the date in a cup and put napkins on top of it to make it clear" (N5).

\section{Patriarchal Family Structure}

Most of the participants indicated that they had difficulty in the initiation of breastfeeding because of elderly family members. A typical participant's statement on this concern was as follows: "... Especially if her mother-in-law or mother are with her, you need to be careful. They may want to learn everything first. They may want to do their own practice. This prevents communication with the mother..." (N6).

\section{Language}

In our study, all of the participants stated that one of the barriers to breastfeeding initiation was the language obstacle. Participants stated that immigrant/foreign mothers didn't know Turkish so they communicated with them using signs and gesture, for example: "...We often cannot communicate with immigrants. No interpreter... We are trying to communicate with them through body language. But how much can you tell with gestures! I can only show the way of breastfeeding. She showed how to hold the breast during breastfeeding on her body with her fingers. But the other parts are missing..." (N9).

\section{Hospital Environment}

The participants stated that the hospital environment was obstructive to breastfeeding because the lack of privacy and a chaotic environment creates barriers to breastfeeding.

\section{Lack of Privacy}

The participants shared their perception that they had difficulties with privacy. Here is a statement on the subject of privacy: "...Sometimes, the mother doesn't want to be talked about too much when her mother-in-law is present. For example, your nipple is too small. The woman feels disturbed when you say that. After all, she is a woman too...." (N10); "...Visitors do not respect the privacy of the patient at the time of breastfeeding. They do not want to leave the room. They say, it is my right..." (N13).

\section{Chaos}

The participants described the first moments after birth as chaotic for the mother: "A baby is crying in her lap... A continuous warning from the surroundings... The doctor says give the baby this. The nurse says do that... relatives say that we did this, we gave water... The mother is not aware of what you are saying or doing. She has just given birth. She has pain. There is a child crying. Everyone is saying something. She feels in the middle of complete confusion, chaos" (N3).

\section{Health Care Staff}

They said that initiating breastfeeding in the early period has some difficulties due to understaffing and employee turnover rates. It was also noted that some of the clinic staff were not sufficiently knowledgeable on the subject of breastfeeding.

\section{Understaffing}

The participants stated that they could not provide the necessary care and support for the initiation of breastfeeding due to understaffing. The workload was one of the obstacles to the early initiation of breastfeeding due to the multitasking structure of their work. Here is a typical comment on the subject of understaffing: "The number of patients is too many. The number of nurses is insufficient... There are both gynecology and maternity patients in our clinic. They also have treatments and follow-ups. We may have to be less concerned with breastfeeding" (N1).

\section{Insufficient Knowledge}

Some of the participants indicated that the healthcare staff did not have enough knowledge about breastfeeding: "The mother's breasts are swollen and stretched. The formula is started because there is no breast milk. Actually, there is breast milk, but the baby does not suck it properly. Unfortunately, both nurses and doctors have difficulties in evaluating breastfeeding..." (N12).

\section{Staff Turnover Rates}

Most of the participants pointed out the negative effects of staff turnover. They stated that they could not always speak a common language with other healthcare staff because of high staff turnover rates and that they had difficulties in the initiation of breastfeeding as a result. According to the statements of the participants, staff turnover leads to a lack of knowledge and the spread of some wrong practices related to breastfeeding. For example: "We may not speak a common language to discuss breastfeeding with other health personnel at the clinic. It is 
caused by very rapid clinical changes of nurses and doctors. Also, different applications are emerging" (N2).

\section{Discussion}

The statistics related to breastfeeding in Turkey show that there are some problems in the initiation of breastfeeding (5). In Turkey, the healthcare staff who are seen first after the mother and baby leave the delivery room and who should support the initial breastfeeding are usually nurses in charge at the maternity clinics (13). It is important to examine in depth the practices of these nurses and the connected influencing factors. Therefore, this study was conducted to define the perceived barriers to the initiation of breastfeeding at the early postpartum period by those nurses working in maternity clinics. The identified barriers perceived as a result of the interviews, analysis and interpretation, were grouped into four themes, namely; delivery, culture, hospital environment and health care staff.

Breastfeeding problems are frequently encountered in the early postpartum period, particularly for women who have had a cesarean birth $(14,15)$. In this study, it was determined that cesarean delivery delayed the initiation of breastfeeding. According to a qualitative study by Majra and Sılan (12), cesarean section causes about a day's delay in the initiation of breastfeeding. Adverse effects of anesthesia on the mother and infant, delayed lactation, maternal discomfort and pain are connected with the effects of cesarean deliveries (16). It has been reported in the literature that mothers who have spontaneous vaginal births have a higher rate of breastfeeding in the first hour than those who have a cesarean delivery $(10,17,18)$. These findings are similar to our study.

Another problem is the perception of insufficient breast milk because the mother feels she does not have enough lactation to meet her baby's needs. This is all related to the mother's perceptions (19). In our study, participants expressed the notion that the perception of insufficient breast milk was an important barrier to the initiation of early breastfeeding, especially in cesarean deliveries. Similarly, it was found that mothers gave their baby supplementary formula in the first 72 hours after birth because of the perception of low milk. This was one of the results of a qualitative study examining the effects of cesarean delivery on breastfeeding (20). According to another qualitative study about barriers to exclusive breastfeeding in American Samoa conducted by Hawley et al. (21), mothers thought that they did not have enough lactation to meet their babies' needs and thus the mothers supplemented their breastfeeding with formula. Nguyen et al. (22) reported that $7.9 \%$ of the mothers included in their study were aware that newborn babies need only 5-7 mL of milk per feed in the first day of life, and half of the mothers felt confident in their ability to provide enough lactation to exclusively breastfeed for the first 24 hours.

The ten steps of successful breastfeeding recommend that newborns are to be given no nutrient or drink except for breastmilk, unless medically indicated otherwise $(1,4)$. Cultural practices that encourage the giving of other liquids instead of breastmilk and pre-lacteal feeding caused a delay for the optimal timing of breastfeeding initiation $(10,12,22-$ 24). Hmone et al. (11) expressed that traditional practices and the intervention of family elders negatively affected breastfeeding. In a qualitative study by Lee et al. (25), they reported that the elders of the family cause a delay in the early initiation of breastfeeding. These outcomes are similar to the findings of our study.

In Turkey, the number of irregular migrants is 174,466 and the number of foreigners holding a residence permit is 461,217 . By the year 2017 , the count of Syrians covered by temporary protection was 3,400,195 and the number of Syrians outside temporary shelters was 3,171,721. Of these, $1,564,897$ are women, and about half of these women are fertile (26). One of the obstacles preventing immigrant women from benefiting from prenatal and postpartum health services is language (27). Similarly, in our study, one of the obstacles encountered by the participants was that they could not communicate with migrant mothers because of language problems.

The other important barrier was privacy. In our study, participants expressed that they had problems with privacy due to crowded patient rooms. Postnatal wards and busy postpartum units are public places for many new mothers where privacy cannot be provided (28). Morrison et al. (29) discovered that mothers try to breastfeed but found their personal boundaries were violated in hospital. According to a qualitative study conducted in Turkey, new mothers had the perception that their privacy was severely curtailed during breastfeeding in a maternity clinic because they were uncomfortable with visitors present and thought that hospitals were not places where personal space was honored (30).

The results of our study, similar to others, confirmed that workload, understaffing and high staff turnover rates have an unfavorable impact on the early initiation of breastfeeding $(12,20,25)$. Moreover, in our study, mothers being given conflicting information about breastfeeding 
was highlighted. Studies have shown that breastfeeding training and counseling given to mothers by healthcare personnel positively affects breastfeeding $(31,32)$. However, the information provided should be consistent. Similar to the findings of other research $(12,20)$, it was determined that conflicting information given to mothers by healthcare personnel was a cause of confusion and led to a failure to initiate breastfeeding by mothers.

\section{Study Limitations}

Because of the qualitative nature of this study, generalizability is low. The results of this study being specific to the limited context, qualitative findings cannot be generalized to all hospitals in Turkey.

\section{Conclusion}

Interviewing nurses early about barriers to the early initiation of breastfeeding provided a deeper understanding of this critical period. As a result of our study, the barriers to the early initiation of breastfeeding were related to factors concerning delivery, culture, hospital environment and health care staff. These barriers can be overcome when pregnant women/new mothers are briefed on the procedure by healthcare staff. For this reason, prenatal education is important in overcoming obstacles related to delivery and culture. Immigrant-friendly hospitals with on-site interpreters are a solution for language-related obstacles. In overcoming the obstacles related to the hospital environment, it is suggested that a positive, calm, privacy-friendly environment be ensured. To increase the competence of health personnel, there is the need for the sufficient number of healthcare staff to be employed and regular training of staff to be given.

\section{Acknowledgement}

We would like to thank the nurses who participated in this study.

\section{Ethics}

Ethics Committee Approval: Approval of the Ethics Committee and permission of the institution were taken (decision no: 60116787-020/29033).

Informed Consent: Verbal and written permission was obtained from the participants before the interviews commenced and were tape-recorded.

Peer-review: Externally peer-reviewed.

\section{Authorship Contributions}

Concept: S.S.C., B.Ç., Design: S.S.C., B.Ç., Data Collection or Processing: S.S.C., B.Ç., Analysis or Interpretation: S.S.C., B.Ç., Literature Search: S.S.C., B.Ç., Writing: S.S.C., B.Ç.
Conflict of Interest: No conflict of interest was declared by the authors.

Financial Disclosure: The authors declared that this study received no financial support

\section{References}

1. World Health Organization. Guideline: protecting, promoting and supporting breastfeeding in facilities providing maternity and newborn services. Geneva: World Health Organization. Licence: CC BY-NC-SA 3.0 ICO. 2017.

2. United Nations Children's Fund (UNICEF). World Health Organization. Capture the moment, early initiation of breastfeeding: The best start for every newborn. New York: UNICEF; 2018.

3. World Health Organization, \& Unicef. Protecting, promoting and supporting breast-feeding: the special role of maternity services. World Health Organization. 1989.

4. Republic of Turkey Ministry of Health. Breastfeeding Counseling Handbook. Ankara: Özyurt Matbaacılık İnş. Taah. San. ve Tic. Ltd. Şti., Ankara, Turkey, 2015.

5. Hacettepe University Institute of Population Studies. 2013 Turkey Demographic and Health Survey. Hacettepe University Institute of Population Studies, T.R. Ministry of Development and TÜBITAK, Ankara, Turkey, 2014.

6. Republic of Turkey Ministry of Health. Health Statistics Yearbook 2017. Kurban Matbaacılık, Ankara, Turkey, 2018.

7. Cangöl $\mathrm{E}$, Şahin $\mathrm{NH}$. Factors affecting breastfeeding and breastfeeding counselling. Med Bull Zeynep Kamil 2014; 45:1005 .

8. Ahmed $A E$, Salih OA. Determinants of the early initiation of breastfeeding in the Kingdom of Saudi Arabia. International breastfeeding journal 2019; 14:13.

9. Creswel JW, Poth CN. Qualitative inquiry and research design: Choosing among five approaches (4th ed.). New York, Sage, 2017.

10. Khanal V, Scott JA, Lee AH, Karkee R, Binns CW. Factors associated with early initiation of breastfeeding in Western Nepal. International Journal of Environmental Research and Public Health 2015; 12:9562-74.

11. Hmone MP, Dibley MJ, Li M, Alam A. A formative study to inform mHealth based randomized controlled trial intervention to promote exclusive breastfeeding practices in Myanmar: incorporating qualitative study findings. BMC Medical Informatics And Decision Making 2016; 16:60.

12. Majra JP, Silan VK. Barriers to Early Initiation and Continuation of Breastfeeding in a Tertiary care Institute of Haryana: A Qualitative Study in Nursing Care Providers. Journal of clinical and diagnostic research: JCDR 2016; 10:16-20.

13. Republic of Turkey Ministry of Health. Postpartum Care Management Guidelines. Ankara, Turkey, 2014.

14. Chien LY, Tai C). Effect of delivery method and timing of breastfeeding initiation on breastfeeding outcomes in Taiwan. Birth 2007; 34:123-30.

15. Zanardo V, Svegliado G, Cavallin F, et al. Elective cesarean delivery: does it have a negative effect on breastfeeding? Birth 2010; 37:275-9. 
16. Dennis CL. Breastfeeding initiation and duration: a 1990-2000 literature review. JOGNN 2002; 31:12-32.

17. Prior E, Santhakumaran S, Gale C, Philipps LH, Modi N, Hyde M). Breastfeeding after cesarean delivery: a systematic review and meta-analysis of world literature. The American Journal of Clinical Nutrition 2012; 95:1113-35.

18. Liben ML, Yesuf EM. Determinants of early initiation of breastfeeding in Amibara district, Northeastern Ethiopia: a community based cross-sectional study. International Breastfeeding Journal 2016; 11:7.

19. Hill PD, Humenick SS. Insufficient milk supply. Journal of Nursing Scholarship 1989; 21:145-8.

20. Chaplin J, Kelly J, Kildea S. Maternal perceptions of breastfeeding difficulty after caesarean section with regional anaesthesia: A qualitative study. Women and Birth 2016; 29:144-52.

21. Hawley NL, Rosen RK, Strait EA, et al. Mothers' attitudes and beliefs about infant feeding highlight barriers to exclusive breastfeeding in American Samoa. Women and Birth 2015; 28:80-6.

22. Nguyen PH, Keithly SC, Nguyen NT, Nguyen TT, Tran LM, Hajeebhoy N. Prelacteal feeding practices in Vietnam: challenges and associated factors. BMC Public Health 2013; 13:932.

23. Patel A, Banerjee A, Kaletwad A. Factors associated with prelacteal feeding and timely initiation of breastfeeding in hospital-delivered infants in India. Journal of Human Lactation 2013; 29:572-8.

24. Sundaram ME, Ali $H$, Mehra $S$, et al. Early newborn ritual foods correlate with delayed breastfeeding initiation in rural Bangladesh. International Breastfeeding Journal 2016; 11:31.
25. Lee HMH, Durham J, Booth J, Sychareun V. A qualitative study on the breastfeeding experiences of first-time mothers in Vientiane, Lao PDR. BMC Pregnancy and Childbirth 2013; 13:223.

26. Republic of Turkey Ministry of Interior. 2017 Migration statistics. Retrieved from https://www.goc.gov.tr/icerik3/gecicikoruma_363_378_4713

27. Aydın R, Körükcü Ö, Kabukçuoğlu K. Transition to motherhood as an immigrant: Risks and obstacles. Current Approaches in Psychiatry 2017; 9:250-62.

28. McInn es RJ, Chambers JA. Supporting breastfeeding mothers: Qualitative synthesis. Journal of Advanced Nursing 2008; 62:407-27.

29. Morrison B, Ludington-Hoe S, Anderson CC. Interruptions to breastfeeding dyads on postpartum day 1 in a university hospital. JOGNN 2006; 35:709-16.

30. Sayın SN. Perception of privacy in perinatal services. University Institute of Health Sciences, Doctor of Philosophy Thesis, isstanbul, 2013.

31. Renfrew M), McCormick FM, Wade A, Quinn B, Dowswell T. Support for healthy breastfeeding mothers with healthy term babies. Cochrane Database of Systematic Reviews 2012; 5.

32. McFadden A, Gavine A, Renfrew MJ, et al. Support for healthy breastfeeding mothers with healthy term babies. Cochrane Database of Systematic Reviews 2017; 2. 